\title{
Approche comparée des processus RSS dans les Grands Lacs
}

Jean-Jacques Patry

\section{(2) OpenEdition}

Édition électronique

URL : https://journals.openedition.org/eastafrica/391

DOI : 10.4000/eastafrica.391

ISSN : 2790-1076

Éditeur

IFRA - Institut Français de Recherche en Afrique

Édition imprimée

Date de publication : 1 avril 2014

Pagination : 107-138

ISSN : 2071-7245

Référence électronique

Jean-Jacques Patry, "Approche comparée des processus RSS dans les Grands Lacs », Les Cahiers d'Afrique de l'Est / The East African Review [En ligne], 48 | 2014, mis en ligne le 07 mai 2019, consulté le 09 décembre 2021. URL : http://journals.openedition.org/eastafrica/391 ; DOI : https://doi.org/ 10.4000/eastafrica.391 


\title{
Approche comparée des processus R.S.S. dans les Grands Lacs
}

\author{
Jean-Jacques Patry
}

\section{Introduction : Les Grands Lacs, laboratoires de la RSS}

La réforme du secteur de la sécurité est un thème pris en compte par la communauté internationale à l'occasion de la transition post-guerre froide, quand les pays d'Europe de l'Est se sont " démocratisés ». Elle s'est poursuivie avec l'expérience européenne dans les Balkans à la suite des guerres de l'ex-Yougoslave (Bosnie-Herzégovine, Kosovo, Macédoine). Après avoir concerné l'Amérique Latine et quelques pays d'Asie (TimorLeste), l'essentiel des programmes s'est concentré sur l'Afrique.

En 2012, selon les Nations Unies, douze programmes sur quatorze concernaient le Continent. La région des Grands Lacs y figure en bonne place comme le montre la carte ci-après (voir page suivante):

Par ailleurs, la présence des ambassadeurs du Conseil de sécurité à la séance de clôture de ces " Concertations » est apparue, sinon comme une approbation explicite du procédé mis en œuvre par Kabila, tout au moins comme une faveur faite au Président qui, depuis la catastrophe électorale de 2011, cherchait désespérément à retrouver un peu de soutien de la communauté internationale.

La victoire militaire obtenue au Nord-Kivu, grâce à l'appui des Tanzaniens et des Sud-Africains de la Brigade d'intervention de la MONUSCO, traduit, elle aussi, un renforcement du Président sur le plan interne ou beaucoup l'accusait de collusion avec l'adversaire ougando-rwandais, comme sur le plan international ou il peut désormais afficher une alliance solide allant de Kinshasa à Dar es Salam en passant par Luanda et Pretoria.

Joseph Kabila qui avait envoyé, en décembre 2012, ses plénipotentiaires à Kampala pour arrêter l'offensive du M-23 et n'avait signé l'Accord-cadre à Addis-Abeba que parce que ce dernier garantissait le déploiement de la Brigade d'intervention dans l'Est du pays, a finalement atteint ses objectifs. Il est à peu près certain que ses priorités vont, désormais, se concentrer sur la prolongation de son pouvoir au-delà de décembre 2016 qui devrait marquer la fin de son deuxième mandat. L'urgence n'est donc plus dans les reformes, mais bien dans la construction d'un consensus en faveur d'une Transition permettant l'extension de son mandat et dans la confection d'un calendrier électoral techniquement inattaquable, mais justifiant lui aussi un report des échéances électorales. 


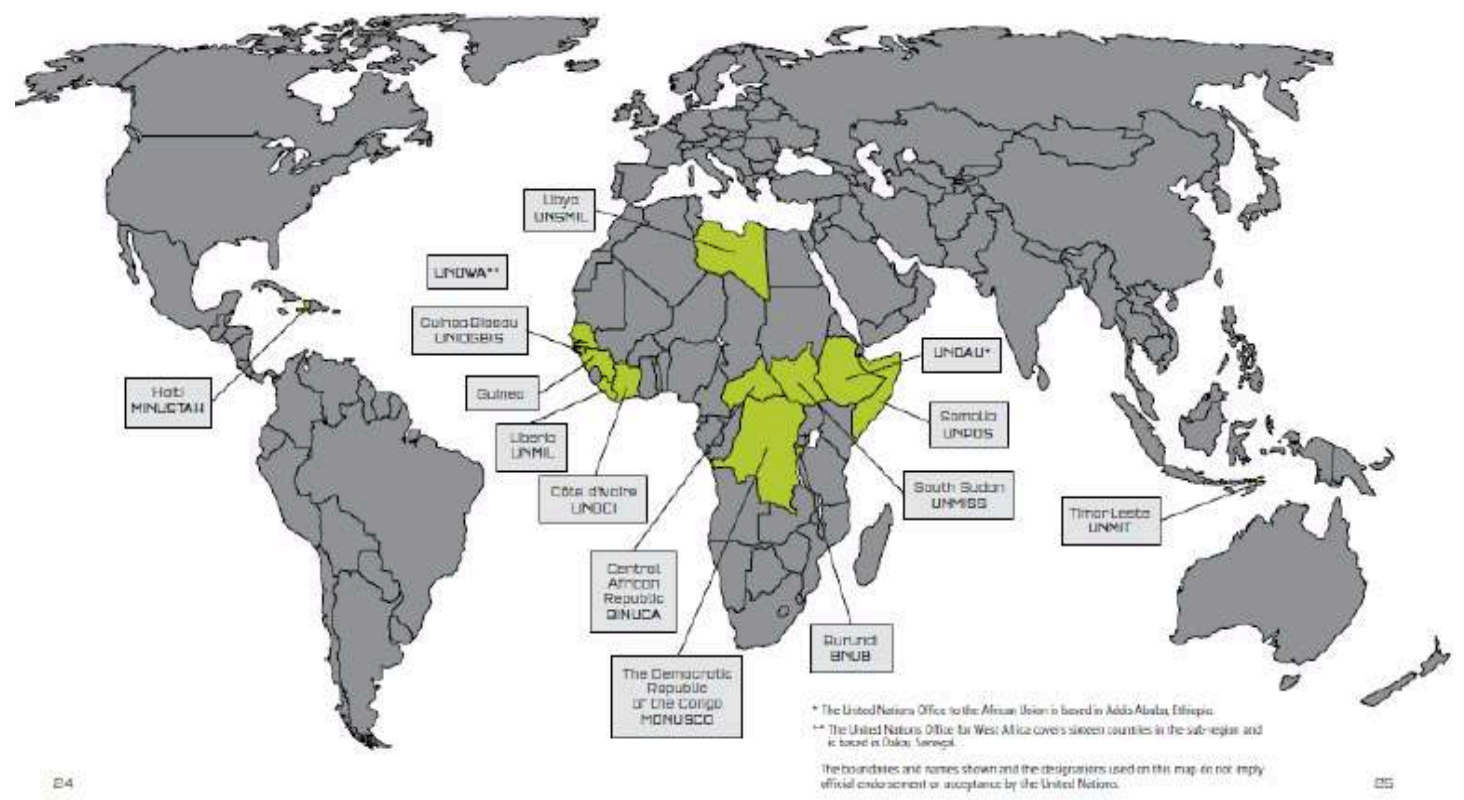

Source : The United Nations SSR Perspective, 2012, pp. 24-25.

A cet égard, il existe huit organisations retenues par le Jane's World Insurgency and Terrorism de 2013 comme suffisamment structurées pour poser des problèmes de sécurité aux pays limitrophes. Certaines sont « endormies » dans le cadre d'un accord de paix, tandis que d'autres développent des stratégies de nuisance autonome ou avec le soutien d'Etats présents dans la zone ${ }^{1}$. Ceci, bien entendu, sans compter les nombreux groupes armés plus ou moins organisés ; simples coupeurs de routes ou milices communautaires locales. La région est donc loin d'être apaisée.

Cette note à pour objet de présenter un point de situation sur les différentes approches de RSS retenues dans les trois pays de la zone faisant encore l'objet d'un programme de réforme : le Burundi, le Rwanda, la République Démocratique du Congo (RDC). Dans le cas de cette dernière, les récents événements intervenus dans les Kivu et la déconfiture institutionnelle nationale rend même difficilement identifiable un vrai processus de RSS.

L'analyse suivra plusieurs étapes :

- Le cadre général de l'exercice de la RSS sur le continent africain sera d'abord précisé. Il existe aujourd'hui une « institutionnalisation » du thème propre au Continent à travers l'Union africaine et son architecture de paix et de sécurité ;

\footnotetext{
1 Au Burundi, les Forces nationales de libération (FNL). Au Rwanda, les Forces démocratiques de libération du Rwanda (FDLR). En République Démocratique du Congo, le Congrès national pour la défense du peuple (CNDP), le Mouvement du 23 mars (M 23), les Patriotes résistants congolais (PARECO). Au Kenya le Sabaot Land Defence Force, en Ouganda, la Lord Resistance Army (LRA), l'Allied Democratic Force (ADF).
} 
- Les principaux acteurs de la RSS opérant dans la zone seront ensuite présentés. Faisant intervenir des institutions internationales, des organismes de conseil et des pays donateurs disposant chacun de leur propre agenda et objectifs. Il est en effet nécessaire de les connaître pour mieux appréhender l'écheveau inextricable qu'ils constituent ;

- Les programmes de réformes seront ensuite analysés pour les trois pays retenus. Pour chaque pays abordé, un éclairage sur la constitution du programme RSS sera présenté. Seront ensuite décrits les axes d'efforts consentis et les acteurs qui prennent place dans le dispositif.

A l'issue de cette analyse, une interprétation est proposée de la portée réelle de la RSS dans la politique de sécurité des pays concernés. Compte tenu de l'extrême complexité de ces dossiers, cette interprétation ne saurait - au mieux - prétendre à autre chose qu'à l'alimentation d'un débat loin d'être achevé.

\section{La RSS en Afrique : un écheveau de responsabilités croisées}

Etablir une perspective aussi claire que possible de la réforme des systèmes de sécurité (RSS) en Afrique des Grands Lacs consiste pratiquement à démêler un écheveau dense de responsabilités croisées et d'initiatives.

Cet écheveau s'explique tout d'abord par le nombre élevé de parties prenantes dans le champ de la RSS : organisations internationales, organisations continentales et régionales africaines, pays donateurs et pays bénéficiaires. Il s'explique aussi par les différents domaines dans lesquels s'exercent les activités de RSS englobant le mode de gouvernance politique ; le fonctionnement des institutions de sécurité militaire, maintien de l'ordre, exercice de la souveraineté territoriale, mais aussi justice et système pénitentiaire. Troisième élément de complexité, la RSS s'applique dans des environnements dégradés : soit des périodes de sortie de conflits armés dans lesquelles il convient de stabiliser de nouvelles institutions, incarnation d'un processus de paix négocié ; soit dans des Etats aux capacités amoindries incapables d'assurer pratiquement la sécurité des citoyens.

En première approche, la RSS est une forme de processus en voie d'appropriation par les organisations du Continent africain, avec l'assistance de nombreuses parties prenantes. La manière dont s'opère cette appropriation collective influence les réussites ou les échecs de ces processus dans les Grands Lacs.

\section{Le cadre d'orientation sur la RSS de l'Union africaine : une appropriation progressive pour l'ensemble des acteurs du Continent}

L'Union africaine est un vecteur de coagulation des différentes activités de la RSS sur le Continent. Elle s'appuie, en partie, sur des capacités détenues par quelques rares organisations régionales comme la CEDEAO. Cette dernière n'entrant pas dans le champ des Grands Lacs, ses capacités ne seront pas présentées. 
Le document de référence dans ce domaine est le Cadre politique sur la réforme du secteur de la sécurité dont l'étude a débuté en février 2008, lors de la dixième session ordinaire de l'Assemblée des Chefs d'Etat et de gouvernements de l'Union africaine. Dans sa version 2013, le « cadre » situe les activités de RSS comme l'un des prolongements concrets de la politique de l'Union sur la reconstruction et de développement post-conflit (RPCD), décidée lors du sommet de Banjul de 2006. Sa rédaction a été largement inspirée par le Réseau africain pour le secteur de la sécurité (RAAS), lui-même conseillé par l'Equipe internationale de conseil la réforme des systèmes de sécurité du Geneva Centre for the Democratic Control of Armed Forces (voir infra).

Il constitue la référence pour l'appropriation par les Africains (Union, architecture de paix et de sécurité, communautés économiques régionales, Etats membres) des principes retenus par les grandes organisations internationales à l'origine du concept de RSS. $\mathrm{RSS}^{2}$ :

L'économie générale du document présente d'abord les acteurs concernés par la

- Les principales institutions de sécurité : militaire, maintien de l'ordre public ; les gardes républicaines ; les services de contrôle et de surveillance des frontières...

- Les institutions spécialisées de renseignement et de sécurité (services de renseignement anti-criminalité, anti-terroriste...)

- Les organismes publics de contrôle et de gestion des institutions précédentes (administrations civiles, parlements...)

- Les institutions chargées de la justice et de l'état de droit

- Les unités civiles d'intervention d'urgence

- Les organes non étatiques de sécurité, telles que les compagnies privées ou les autorités informelles et coutumières

La société civile : un ensemble d'acteurs clés pour la réussite des programmes de RSS

Le " cadre " insiste sur l'importance de la société civile dans l'appropriation réelle de la RSS. Il rappelle donc la définition retenue par l'UA de ses composants. ${ }^{3}$. Il s'agit des groupes sociaux (femmes, enfants, jeunes, personnes âgées, les personnes handicapées..) ; des groupes socioprofessionnels (artistes, ingénieurs, journalistes, enseignants, juristes, théoriciens des affaires sociales, universitaires, commerciales...) ; les organisations non gouvernementales, bénévoles ; les organisations culturelles. Les groupes des diasporas sont aussi inclus dans cette liste.

Très concrètement, la présence en nombre ou non de ces différents leviers d'opinion et de relais de pouvoirs dans la société peut constituer un indicateur de maturité pour évaluer l'opportunité et les chances de succès d'un programme de RSS.

2 Commission de l'Union africaine, Cadre d'orientation sur la réforme du secteur de la sécurité, Addis-Abeba, 2013, 35 p., pp. 7-8.

3 Statuts du Conseil Economique, Social et Culturel de l'Union Africaine (ECOSOCC), article 3. 
La RSS y est définie comme : « le processus par lequel les Etats formulent ou réorientent les cadres institutionnels, les structures et les capacités des institutions et des groupes engagés dans le secteur de la sécurité, en vue de les rendre plus efficaces et de leur permettre d'être réceptifs au contrôle démocratique et attentifs aux besoins de sécurité et de justice de la population $»^{4}$. Cette définition est conforme à celle retenue par les Nations unies $^{5}$.

Le document s'attache ensuite à présenter l'organisation « africanisée » proposée aux acteurs continentaux de la RSS.

La section B présente les «principes africains de base » qui s'ajoutent aux dix règles reconnues par les organisations internationales pour une bonne appropriation ${ }^{6}$ :

- Solidarité et partenariats africains en se servant des possibilités offertes par l'Acte constitutif de l'Union;

- La RSS est reconnue comme un vecteur de l'intégration régionale par le biais de la sécurité assise sur une bonne gouvernance ;

- Tout processus de RSS doit être basé sur l'approbation nationale et faire l'objet d'un engagement national ; lequel doit servir de guide pour l'appui extérieur et permettre aux grands opérateurs de s'adapter à chaque contexte local ;

- A cet effet, l'intégration des institutions informelles et coutumières de sécurité et des acteurs de la justice traditionnelle au sein des processus de la RSS est recommandé ;

- La RSS doit faire partie d'un effort de réforme et d'une démocratisation élargie ;

- Elle doit souscrire aux principes de base de la bonne gouvernance (État de droit, transparence...);

- Elle adhère aux principes de l'égalité des sexes et de la promotion de la femme (la décennie 2010-2020 est dédiée par l'Union à la Femme africaine).

La section $\mathrm{D}$ rentre dans les détails de la conception et de la planification générique des programmes de RSS. La logistique, en quelque sorte, par la structuration de fonctions nationales sur lesquelles devraient venir se greffer l'assistance étrangère :

\footnotetext{
4 Cadre d'orientation sur la réforme du secteur de la sécurité, op. cit., p. 6.

5 UN DPKO, Security Sector Reform Unit : «A process of assessment, review and implementation as well as monitoring and evaluation led by national authorities that has as its goal the enhancement of effective and accountable security for the State and its peoples without discrimination and with full respect for human rights and the rule of law », The United Nations SSR Perspective, , 2012, 52 p., p. 2.

6 Cadre d'orientation sur la réforme du secteur de la sécurité, op. cit. pp. 9-11.
} 
- La fonction évaluation des besoins ;

- La fonction formulation d'une stratégie nationale de RSS ;

- La fonction contrôle du processus de RSS, mais aussi des institutions réformées dans leur fonctionnement quotidien. C'est ici que sont sollicités les organismes parlementaires, d'inspection générale des administrations, du pouvoir judiciaire.

Deux points d'importance critique sont particulièrement soulignés : tout d'abord celui de la formation d'une expertise idoine pour les personnels engagés dans les processus complexes de la RSS. Celle-ci est souvent fournie par l'étranger et tend à se réduire ou disparaître après leur désengagement. La mise sur pied, en parallèle du Réseau africain pour le secteur de la sécurité est une conséquence de ce constat.

\section{Le réseau africain pour le secteur de la sécurité (RASS)}

Le RASS est créé depuis 2003 au Ghana, pays qui abrite son siège. Sa mission principale consiste à conseiller la transformation du secteur de la sécurité et à promouvoir la paix en étoffant les savoir-faire des nombreux organismes africains impliqués dans les processus de RSS. Ses activités se déploient dans trois domaines : la diffusion de la connaissance de la RSS dans un large public et auprès des décideurs politiques du Continent; un soutien technique dans les études de développement institutionnel et des politiques de sécurité; des formations pour les acteurs de sécurité et les organes de surveillance et de contrôle de ces secteurs.

Le RASS souhaite développer une authentique pratique africaine des mécanismes RSS et conseille en ce sens la Commission de l'Union africaine par le biais d'un protocole permanent. Depuis sa création, le réseau développe des partenariats panafricains (African Security Dialogue and Research du Ghana, le Centre pour la démocratie et le développement du Nigeria, le Réseau de gestion de la sécurité et de la défense en Afrique australe, le Centre for Policy Research and Dialogue du Sud-Soudan), le Centre de recherche et d'information sur la sécurité du Kenya). Le réseau est devenu le point de contact privilégié de l'ensemble des organisations internationales impliquées dans les activités de RSS en Afrique.

Dans la zone des Grands Lacs le RASS s'est engagé dans les différents processus de réforme de la police en République démocratique du Congo et au Kenya.

Le second point est d'ordre financier. Le « cadre » propose donc ce qui paraît être l'esquisse d'une «stratégie génétique » de soutien aux activités de RSS. Il recommande un financement croisé des programmes, avec une partie assurée par l'Etat bénéficiaire soutenu, si nécessaire, par le Fond de la paix de l'Union africaine, en complément des financements internationaux. Il recommande ensuite la mise en place et l'application d'un système de financement permanent des institutions de sécurité ainsi renforcées, amenant nécessairement de meilleures pratiques d'audit financières et fiscales.

Depuis janvier 2013, l'African Union Disarmament, Demobilization and Reintegration Capacity Program (AU-DDRCP) est activé au siège de l'Organisation pour prendre en compte l'ensemble des activités de conseils, d'études et de soutien aux Etats membres liées au domaine DDR, première étape du processus SSR. Cette initiative 
lancée par la Banque mondiale et les Nations unies autorise désormais une première institutionnalisation de la question au sein de l'architecture de paix et de sécurité de l'Union africaine ${ }^{7}$.

\section{Soutenue par un maillage dense d'expertise internationale}

Le concept de réforme du secteur de la sécurité (RSS) est lié depuis sa création à l'idée que la sécurité est la condition première favorisant le développement économique. Il concerne les Etats en transition démocratique (pays de l'est-européen post-soviétique et balkaniques, d'Amérique latine), des sociétés émergeant d'une guerre civile (Sierra Leone, Libéria, Ouganda...) ou des Etats dits « faibles » (RDC, Burundi...). Mais, ses concepteurs sont Occidentaux. Il s'agit donc d'une importation sur le continent par le biais d'un réseau extérieur d'assistance technique, dont le maillage s'est progressivement mis en place par le biais d'expériences empiriques et de réflexions de type « retour d'expérience ». Il n'est donc pas inutile de rappeler en quelques lignes qui sont ces grands opérateurs et comment chacun souhaite se placer à l'égard des acteurs locaux de la RSS.

\section{L'Organisation pour la Coopération et le Développement Economique (OCDE)} est la première institution internationale à faire le lien entre sécurité et développement et relier l'ensemble au domaine du rétablissement de la paix. Au sein de l'OCDE, le Comité d'aide au développement (CAD) produit un premier rapport en $1997^{8}$, qui servira de socle pour de nombreux travaux ultérieurs. L'implication d'une large communauté d'acteurs de sécurité, dépassant les forces armées ou de police y est déjà mentionnée. Un groupe de travail sur les conflits, la paix et la coopération pour le développement (CPDCC) produit en 2004 un fascicule sur les lignes directrices applicables à la RSS ${ }^{9}$, puis un manuel complet en 2007 expliquant l'articulation pratique des programmes ${ }^{10}$. Parallèlement aux recherches sur la limitation des violences armées, les analystes de l'OCDE ont approfondi les relations entre les processus de RSS et les outils de réduction de la violence. Une partie des cas étudiés concerne les Grands Lacs ${ }^{11}$.

L'ONU, par le Conseil de sécurité, se saisi du dossier par une déclaration du 27 février 2007 mentionnant l'intérêt d'entreprendre des programmes RSS en vue de prévenir la reprise de conflits armés et de contribuer au maintien et consolidation de la paix. Mandat est confié au Secrétaire général pour l'éclairer. Le rapport rendu en $2008^{12}$ jette un premier dispositif de l'organisation pour traiter du sujet et déterminer les principes cardinaux à mettre en œuvre. Les organes compétents sont la Division des opérations de maintien de

\footnotetext{
7 S.a., The AU-DDRCP, Assisting DDR Processes across the Continent, African Union Peace \& Security Department, 2013.

8 OCDE, Lignes directrices du CAD sur les conflits, la paix et la coopération au développement, Paris, 1997. Réédition 2001. 9 OCDE, Réforme de systèmes de sécurité et gouvernance : principes et bonnes pratiques, Paris, mai 2004. 10 Manuel de l'OCDE sur la réforme des systèmes de sécurité : soutenir la sécurité et la justice, Paris, 2007.

$11 \mathrm{OCDE}$, Relier la réforme des systèmes de sécurité et la réduction des violences armées, note de programmation, Paris, $56 \mathrm{p}$. 12 Securing peace and development: the role of the United Nations in supporting security sector reform, Report of the Secretary general, A/62/659-S/2008/39, 23 January 2008, 19 p.
} 
la paix, impliquée de longue date dans les programme de désarmement, démobilisation, réintégration (DDR) avec, au sein du Bureau de l'état de droit, l'Unité de réforme du secteur de la sécurité. Cette dernière, codirigée par le Programme de développement des Nations Unie constitue le « centre d'excellence » et le point de contact et de coordination inter-organisationnel pour les activités RSS de l'ONU. C'est par son biais que sont organisées les activités vers les organisations régionales de sécurité comme l'Union Africaine et ses Etats membres. Il convient d'y ajouter les travaux de la Commission de consolidation de la paix (CCP) qui, depuis 2005, assure une fonction de conseil pour les « sorties de crise ». Six pays africains sont en 2013 à l'ordre du jour : Sierra-Léone, Libéria, les deux Guinées, la République centrafricaine et le Burundi.

L'Union européenne a engagé une action globale dans le domaine de la RSS au titre de sa stratégie générale de sécurité de 2003. Le cadre d'ensemble est constitué par le concept de l'UE pour le soutien de la PESC à la RSS, d'octobre 2005 produit par Comité politique et de sécurité et par la communication Réflexion sur l'appui apporté par la Communauté européenne à la réforme du secteur de la sécurité ${ }^{13}$. L'expérience technique civile, policière et réforme de la gouvernance s'est forgée dans les Balkans, puis s'est étendue à l'Afrique, notamment, avec les missions en Guinée Bissau, Somalie et surtout en RDC (EUSEC RD Congo et EUPOL RD Congo). L'importance de la RSS est régulièrement rappelée comme une priorité d'action de la stratégie de l'Union ${ }^{14}$.

L'Organisation internationale de la Francophonie (OIF) assure un rôle plus indirect de conseil concernant l'environnement « bonne gouvernance », depuis 2008, qui devrait faciliter l'appropriation locale des programmes de RSS. Agissant avec les autres pôles de compétences, l'OIF se spécialise dans l'information et l'éducation des fonctionnaires et agents publics de maintien de l'ordre et de la justice concernant les droits des individus, les normes institutionnels à développer et la connaissance croisée du fonctionnement de ces institutions. L'OIF insiste aussi sur les techniques de gestion et de contrôle civil et parlementaire des institutions de sécurité. Un document de référence paru en 2010 présente la vision des pays francophones sur l'appropriation pratique d'un concept anglo-saxon ${ }^{15}$. L'essentiel des cas traités concerne l'Afrique francophone.

La Banque Mondiale (BM) et le Fond Monétaire international (FMI) ${ }^{16}$ jouent un rôle de conseil financier et reflètent, en outre, les priorités désignées par les Etats donateurs dans la structuration du financement des programmes de développement. Ils travaillent en étroite collaboration avec les organisations en charge de la réalisation des programmes RSS.

13 Réflexion sur l'appui apporté par la Communauté européenne à la réforme du secteur de la sécurité, communication de la Commission au Conseil et au Parlement européen, Bruxelles, 24 mai 2006, 14 p.

14 Rapport sur la mise en cuvre de la stratégie européenne de sécurité : assurer la sécurité dans un monde en mutation, S407/08, 11 décembre 2008, 12 p., pp. 7-8.

15 OIF, La réforme des secteurs de la sécurité et de justice en Afrique francophone, Paris, 2010, 319 p.

16 Ball, N. World Bank/IMF: Financial and Programme Support for SSR. 
Au sein de la BM, la question de la RSS est prise en compte :

- Au titre des «stratégies de réduction de la pauvreté », à travers des prêts et les financements d'institutions (Capacity Building) dans des Etats dits faibles ;

- Au titre la consolidation des pays sortant de conflits armés par une politique particulière de financements dont la rationalité est présentée dans un document spécial de référence : Operational Policy 2.30 " Development Cooperation and Conflict ». L'intégration des mécanismes de RSS y est particulièrement étudiée.

Le FMI, pour sa part, a développé un code de bonnes pratiques et de responsabilités fiscales pour améliorer les capacités de transparence et de lutte anti-corruption ; environnement dans lequel les initiatives RSS peuvent plus facilement aboutir. L'action de ces deux institutions permet concrètement de financer une expertise applicable aux processus RSS et DDR, dont l'ISSAT offre un bon exemple. L'Association internationale de développement (IDA), le fond d'intervention de la BM, sert de vecteur financier (Burundi).

International Security Sector Advisory Team (ISSAT) ou Equipe internationale de conseil au secteur de la sécurité a été créée en 2008 au sein du Geneva Centre for the Democratic Control of Armed Forces. L'organisation est conçue comme un réservoir d'expertise globale incluant les quatre phases génériques d'un programme de RSS : la formulation initiale des besoins ; la planification ; la mise en œuvre ; la surveillance et l'évaluation des résultats. Ce cycle s'applique dans tous les domaines des secteurs de sécurité (militaire, police, justice...), lesquels sont travaillés au sein d'une Communauté RSS rassemblant les institutions déjà précitées et des Etats bénéficiaires ${ }^{17}$.

Les donateurs nationaux. Ceux-ci étant nombreux, avec des niveaux d'engagement très divers, seuls ceux impliqués dans les programmes de la région des Grands Lacs seront abordés. Toutefois, il convient de noter qu'en l'absence de ces collaborations bilatérales, de nombreux programmes seraient simplement dépourvus de substance.

\section{Le socle de la RSS dans les Grands Lacs : un processus DDR réussi ou non}

La réforme du secteur de la sécurité pose donc, comme condition sine qua non du succès, une situation de départ suffisamment calme pour permettre aux nombreuses parties prenantes d'intervenir sur zone et de déployer les équipes et capacités nécessaires. Ceci implique pour les Grands Lacs, sortant de deux décennies de guerre, l'application d'accords DDR, première étape de réalisation d'un processus de paix. 
Cette étape a été engagée entre 2002 et fin 2009. Le plus grand programme de réforme du secteur de la sécurité que la communauté internationale n'ait jamais lancé, s'est focalisé sur la région africaine des Grands lacs.

Table 4: National Programs and Special Projects Funded by the MDRP and IDA

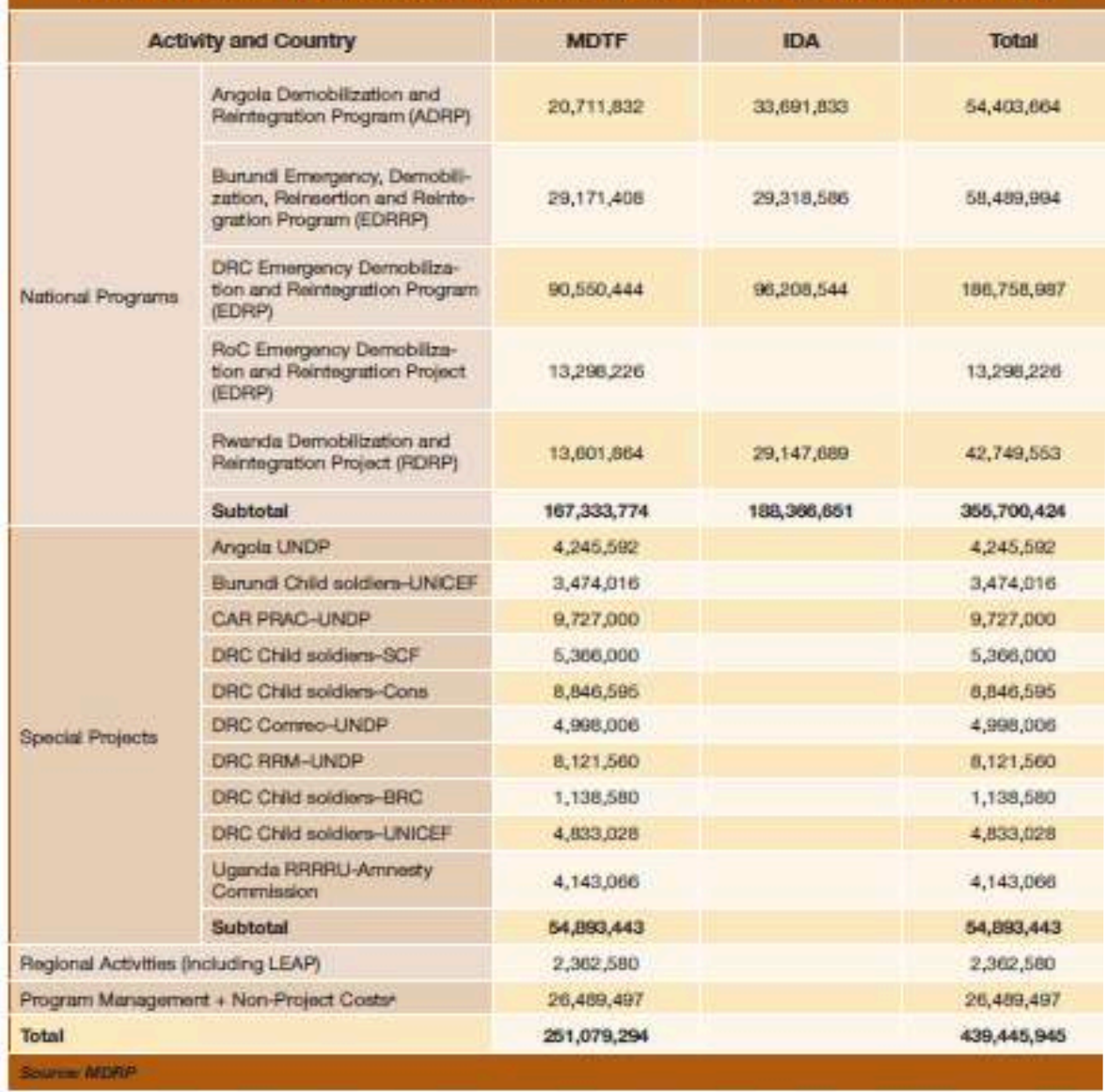

" Non-Project Costs refer to the Bank's administration tees for managing the Multi-Donor Thust Fund, based on co tributions recaived from donors.

Source : MDRP Final Report: Overview of Program Achievements, p. 22 . 
Le Multi-Country Demobilization and Reintegration Program (MDRP) a rassemblé 13 pays donateurs avec le concours de la Banque mondiale (programme IDA), 21 organisations internationales relevant des Nations unies et régionales appartenant à l'Union africaine, pour assister 7 pays de la zone et de son proche voisinage : Angola, Burundi, République du Congo, République Démocratique du Congo, République de Centre-Afrique, Rwanda, Ouganda. Avec un budget dépassant les 500 millions de dollars (2009), le programme a permis de démilitariser près de 350000 ex-combattants, d'en réintégrer 2800000 , dont environ 53000 enfants-soldats ${ }^{18}$. Le tableau suivant présente l'architecture d'ensemble.

Le MDRP offre un bon exemple de la complexité de ces programmes, en termes du nombre d'acteurs impliqués de nature étatique, internationale, institutionnelle ou provenant de la société civile. L'architecture se composait de sept programmes nationaux et de dix projets spéciaux, dont il a fallu coordonner les différents aspects.

Il illustre parfaitement la dépendance des activités de démilitarisation d'une région (désarmement, démobilisation, réintégration) à l'égard de la réussite du processus de paix politique qui sert de cadre et des progrès socio-économiques de développement, autorisant ou non la réintégration durable des ex-militaires dans la société civile.

Les retours d'expérience ont fait l'objet d'une littérature abondante ${ }^{19}$. Un audit indépendant à été réalisé à la clôture du programme en juin $2009^{20}$. Les points essentiels suivants ont été retenus pour accroître les chances de succès d'une telle entreprise dans le futur :

- L'appropriation nationale du programme par chaque Etat bénéficiaire est indispensable. Elle doit toutefois s'accompagner d'un engagement lisible des partenaires extérieurs dans le suivi des actions locales ;

- La réintégration des ex-combattants doit être prévue très en amont des actions de démobilisation, en raison du temps et de la gestion des moyens de préparation de l'insertion dans le tissus socio-économique ;

- Les ex-combattants ne sont pas homogènes. Certains groupes peuvent requérir des capacités de traitement différencié. Si le groupe des enfantssoldats a été très tôt identifié avec la mise au point de projets spécifiques, la question des démobilisés malades a été sous-estimée, de même que la condition d'extrême précarité des femmes ex-combattantes. L'introduction d'une dimension « genre » dans les futurs programmes est recommandée.

18 MDRP Final Report: Overview of Program Achievements, July 2010, 84 p.

19 Le site du MDRP est encore accessible à cette adresse (http://www.mdrp.org/). Il propose plusieurs dizaines de monographies et de notes de diffusion regroupées par pays et par thèmes.

20 S.a., Multi-Country Demobilization and Reintegration Report: End of Program Evaluation, Final Report, Scanteam, Oslo, June 2010, 152 p. 
- Il existe de facto une inégalité de situation parmi les Etats bénéficiaires faibles / fragiles ou émergeant d'un conflit armé. Comme les capacités locales sont inexistantes (souvent faute d'administration décente), ces dernière doivent être créées de toute pièce par les partenaires extérieurs ;

- La coordination est critique. Elle implique donc un partage clair des responsabilités de chacun et une fonction " évaluation » permanente et collective de l'efficacité des actions. Les auteurs, sans l'écrire, retiennent la nécessité d'une stratégie intégrale équivalente de la Comprehensive Approach des missions multidimensionnelles de maintien de la paix.

Le Transitional Demobilization and Reintegration Program (TDRP) a été mis en place pour quatre ans (2009-2013), afin d'achever le cycle précédent du MDRP et de poursuivre des échanges d'expériences indispensables à la constitution de capacités DDRSSR autonomes. Le TDRP est d'ailleurs conçu comme un centre d'excellence pour le conseil et la diffusion des bonnes pratiques entre les sept Etats bénéficiaires du MDRP. Le programme peut aussi fournir une assistance financière additionnelle pour certaines initiatives de démobilisation - réinsertion. Le Rwanda dispose, par exemple, d'une contribution de 4,5 millions de dollars pour son programme DDR ${ }^{21}$. Toutefois, le fond alimenté par les donateurs, dont la liste apparaît ci-dessous, ne dépasse pas 33,2 millions de dollars. Il n'autorise donc pas de financements d'envergure.

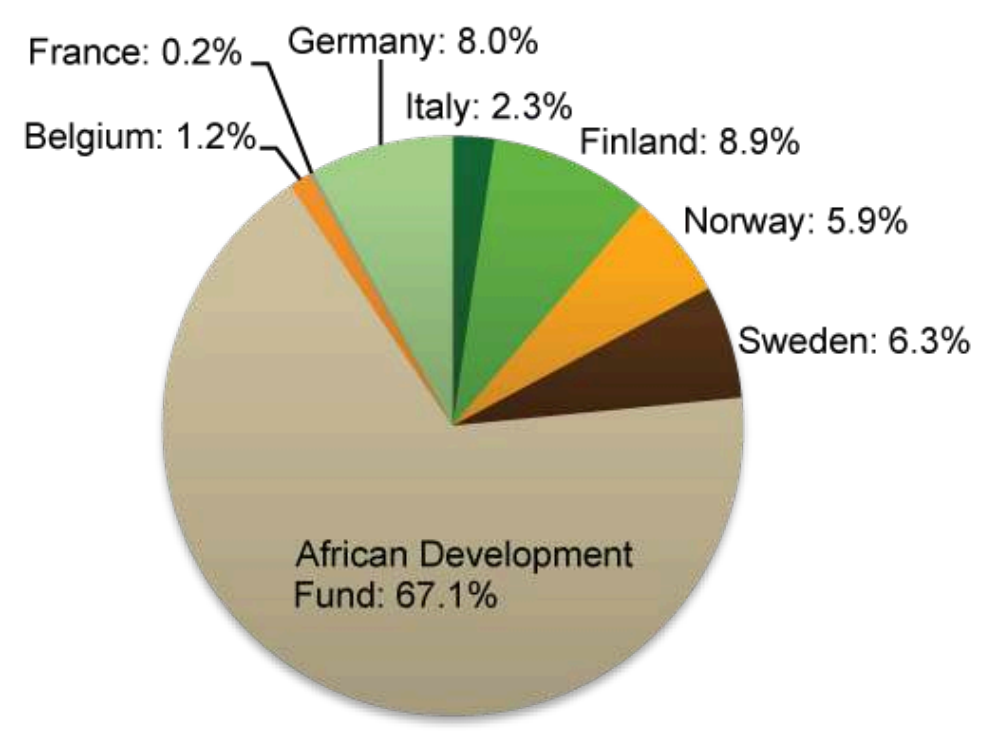

Source : http://tdrp.net/en/donors.html

21 Transitional Demobilization and Reintegration Program at a Glance, November 2011, 3 p., p. 1. 
Le programme se concentre plus pratiquement sur la création et l'animation d'un réseau régional baptisé DDR-Net à partir duquel s'organisent : des échanges régionaux sur l'avancement et les modalités pratiques des programmes DDR ; des forums d'expertise thématiques; des activités de formation techniques et de rencontres ${ }^{22}$.

Pour la région des Grands Lacs, le bilan des différents processus de DDR peut, en 2013, s'établir de la manière suivante :

- Burundi : il reste un programme résiduel de DDR cofinancé par la Banque mondiale et quelques donateurs à partir d'un fond de 10 millions de dollars (voir infra pour plus de détails) :

- Rwanda : une contribution de 4,5 millions de dollars est accordée pour achever un programme considéré comme une vraie réussite (voir infra pour plus de détails).

- Ouganda : les activités de DDR sont closes depuis 2011.

- République Démocratique du Congo : depuis 2004, les activités DDR ont impliqué la démobilisation de 109846 combattants (dont 31378 enfantssoldats) et l'assistance à la réintégration de 83360 démobilisés. Une structure permanente, le programme national de désarmement, démobilisation et réintégration est créée depuis 2007. Les financements internationaux sont clos depuis 2011. Toutefois, le pays est encore en guerre dans l'Est et l'intégration des ex-combattants est dictée par les aléas du soutien des pays voisins à de nouveaux groupes rebelles.

En 2013, on peut donc considérer, sauf pour la RDC, que la phase DDR étant achevée ou en bonne voie, la réforme du secteur de la sécurité dans les autres domaines peut s'amorcer. C'est vrai pour le Burundi et le Rwanda.

\section{Le Burundi : la RSS est le cour de l'Accord d'Arusha}

Le Burundi offre l'exemple d'un pays polarisé sur une répartition équitable du pouvoir entre une élite minoritaire tutsie et une majorité hutu insatisfaite de son sort. Dans un tel contexte, le contrôle des appareils de sécurité est une question cruciale pour la survie de chaque camp. La question de la représentation des différentes composantes communautaires dans ces institutions a constitué un enjeu dans le conflit interne, puis dans le processus de paix qui sert de cadre général à la RSS. Cet accord, fruit d'une longue négociation entre les factions, a suivi le remplacement progressif de l'élite Tutsie par des partis politiques Hutus au fur et à mesure de leur intégration dans le jeu politique.

22 L'ensemble des activités du TDRP sont présentées dans, Transitional Demobilization and Reintegration Program for the Great Lakes Region: Program Paper and Operational Procedures, November 2009, 42 p. 
Le programme de RSS au Burundi constitue un effort clé des Accords de paix d'Arusha, à la suite de la dernière guerre civile dont le pays est victime entre 1993 et 2000. Il prend racine dans le chapitre 2 du protocole III du document ${ }^{23}$ et son application, très progressive, suit les aléas de l'application d'Arusha. Les processus de réformes concernent les Forces de Défense Nationale (FDN) et la Police Nationale du Burundi (PNB). La Justice est le troisième secteur clé du programme avec la question de la gestion de la justice transitionnelle.

\section{Réformes des FDN : démobilisation - bureaucratisation}

Intégré au processus d'institutionnalisation politique, le cadre de la RSS est prévu par la constitution adoptée en 2005 qui dispose de la création d'un Conseil national de la sécurité présidé par le Chef de l'Etat. Le Conseil entre en fonction à partir de $2008^{24}$. Toutefois, les activités concrètes de réformes s'engagent au fur et à mesure que s'engrangent des accords de cessez-le feu entre les protagonistes. Ces derniers s'opèrent sur plusieurs années :

- En 2000, une partie des groupes armés hutus (le G7), s'associe aux Forces armées du Burundi (FAB) et à leurs groupes alliés tustie (G-10) pour signer les Accords d'Arusha. Mais les principales organisations hutus rebelles sont encore absentes ;

- En 2003, un cessez-le feu avec le Conseil national pour la défense de la démocratie - Forces pour la Défense de la Démocratie (CNDD-FDD) permet d'amorcer son processus d'intégration politique et d'entamer la démobilisation des combattants. Une masse critique est atteinte pour lancer le programme ;

- En 2006, un autre cessez-le feu intervient avec le Parti pour la libération du peuple hutu - Forces nationales de libération (PALIPEHUTU - FNL), dernière grand organisation armée à accepter l'Accord de paix.

Deux ensembles de dispositifs sont mis en œuvre pour engager la réforme des armées et celles de la police. Ceux-ci seront soutenus par le Bureau intégré des Nations unies au Burundi (BINUB) qui s'ouvre en 2006 et devient, en 2011, le Bureau des Nations Unies au Burundi (BNUB).

23 Accords d'Arusha pour la paix et la réconciliation au Burundi, 28 août 2000, 180 p., pp. 64-76.

24 Mora, S. La réforme du secteur de la sécurité au Burundi, Initiative pour la construction de la paix, Union européenne - CENAP, novembre 2008, 30 p., pp 14-15. 


\section{Un observateur engagé : le Centre d'alerte et de prévention des conflits - CENAP}

Le CENAP est une association à but non lucratif, créée en 2004. Elle s'est fixée comme objectif de contribuer au dialogue entre les différents intervenants du processus politique burundais et la société civile sur les thèmes suivants : états fragiles et consolidation de la paix, suivi et évaluation des processus électoraux, justice transitionnelle, question de pauvreté, chômage et sous-emploi. Les questions du désarmement des civils et de la RSS occupent une place importante. A ce titre, elle a réalisé plusieurs études sociologiques sur les différentes perceptions de la réinsertion des anciens combattants et sur la manière dont la population considère les institutions de sécurité (armée et police). Le CENAP fait appel à une douzaine de spécialistes indépendants et s'attache à intégrer une dimension " genre » dans ses analyses.

S'agissant de la conclusion d'une guerre civile d'origine communautaire, le principe directeur de la réforme consiste à appliquer une politique de représentation équilibrée des communautés dans le système de pouvoir. Cela revient à instaurer une politique de quota dans les institutions de sécurité et à les « dépolitiser » par une "professionnalisation technique ». Les Accords d'Arusha prévoient donc qu'aucune communauté ne peut investir plus de $50 \%$ des institutions de sécurité. Les membres de ces nouvelles institutions doivent devenir des professionnels et s'abstenir de toute activité politique; ils doivent obéissance au gouvernement central et au Parlement ; enfin, les auteurs de crimes de guerre ou de masse sont écartés du bénéfice de la réintégration dans les forces armées ou de la police réformée.

Le démarrage à lieu début 2003, avec la création d'une Commission nationale pour la démobilisation, réinsertion et réintégration (CNDRR), chargée de mettre en œuvre le programme DDR définit par le gouvernement de transition. Il sera ensuite épaulé, après l'inclusion du CNDD-FDP dans le processus électoral de 2005, par le nouvel état-major des FDB. Cet organisme, dirigé par deux commandants autrefois ennemis : les généraux de brigade Germain Niyoyankana (ancien FAB) et Adolphe Nshimirimana, son adjoint (ancien CNDD-FDD) assure les études et arbitrages techniques du processus de démobilisation des anciens combattants, baptisés PMPA (partis et mouvements politiques armés).

Concrètement, il s'agit de se séparer d'une partie importante des ex-Forces armées du Burundi et de recruter des anciens rebelles opposants hutus. D'après les chiffres présentés par les Nations unies, la phase initiale de désarmement, démobilisation concerne, entre 2004 et 2008, 43000 personnels des ex-FAB et 21000 irréguliers pour ne retenir que 25000 personnes dans l'enveloppe fixée ${ }^{25}$.

En 2006, après le ralliement du PALIPEHUTU - FNL, il convient d'ajouter le désarmement de 2500 hommes supplémentaires. In fine, 900 seront retenus pour grossir les rangs de la nouvelle armée, en 2009.

25 Security Sector Reform Monitor, Burundi, nº4, October 2010, 12 p., p. 4. 
Deux phases sont identifiables : la première concerne un processus tout à fait classique de désarmement, démobilisation et réinsertion des ex-combattants. Puis, à partir de 2006, une « bureaucratisation » est mise en place avec un effort important « d'encasernement » des personnels pour gérer les processus de formation et de réintégration.

La mise sous contrôle et la bureaucratisation des personnels favorisent l'installation d'organes de contrôle des nouvelles institutions :

- L'équivalent d'une Cours des comptes s'assure de la bonne exécution de la procédure budgétaire. La lutte contre la corruption devient ainsi plus crédible ;

- Un corps d'inspection générale est créée avec un système judiciaire militaire fixe et itinérant pour juger rapidement des actes d'indisciplines et des crimes ;

- Le Parlement créée dans chacune des chambres une Commission défense et sécurité qui, peu à peu, commencent à mener des analyses et réalisent des enquêtes au sein des forces armées.

C'est ainsi qu'à partir de 2008, le Sénat publie le premier rapport sur l'application des quotas au sein des armées. Il en ressort que 30,2\% des postes de commandement étaient à l'époque détenus par des Hutus; que les cadres tutsis restaient majoritaires dans les unités spécialisées. Il était recommandé d'accélérer la féminisation de l'armée pour faciliter la transition vers les 50\% fixés par les Accords d'Arusha. Toutefois, le rôle de ces organes de contrôle est difficile à maintenir en fonction. Ils sont en effet anémiés par les difficultés de marchandages politiques qui, en permanence, rythment l'application quotidienne des Accords d'Arusha.

\section{La réforme de la police : unification - civilianisation}

Simultanément, le travail de réforme de la police et de la justice a suivi un parcours une peu différent ${ }^{26}$. Le pays, à la suite de la guerre civile, a connu une situation sécuritaire intérieure aggravée par :

- La circulation des armes dans la société et l'habitude de les utiliser pour régler toute forme de conflit personnel ou d'intérêts ;

- La violence des anciens combattants non ou mal intégrés et qui versent dans la criminalité de droit commun ;

- L'impunité liée à l'absence de service public de justice, facilitant les attaques contre des minorités ethniques ou contre des personnes plus vulnérables (femmes) ;

- L'utilisation du pouvoir en place des organes de sécurité pour réprimer toute opposition légale.

26 Nindorera, W. La réforme du secteur de sécurité au Burundi : enjeux et défis pour une meilleure protection des populations civiles, CENAP, INS, Bujumbura, juillet 2007, 52 p., p. 19. 
Les Accords d'Arusha ont prévu l'unification de différents corps de police. Le 31 décembre 2004, la police nationale du Burundi rassemble des effectifs de l'ancienne police judiciaire, de la police de sécurité publique, de la police de l'air, des frontières et des étrangers. S'y adjoignent des anciens de la Gendarmerie, des forces armées et des différents groupes combattants. Le résultat a été une « militarisation » du corps de police à près de 89\% des nouveaux effectifs (18 164 en 2009), rendant problématiques les relations avec le public ${ }^{27}$. Cela s'est traduit par l'utilisation disproportionnée de la force dans les missions de maintien de l'ordre, des abus d'autorité, voire des comportements délictueux des policiers (200 personnels accusés et arrêtés en 2009). Le manque de moyen pour l'entraînement et l'équipement expliquent cette situation.

Contrairement à l'armée qui doit être encasernée pour assurer son contrôle, les efforts de modernisation de la police doivent viser en priorité une « démilitarisation des cultures opérationnelles des personnels $»$.

\section{La réforme judiciaire : l'improbable justice transitionnelle}

Le système policier est d'autant plus critiqué que les débordements qu'il occasionne ne peuvent être sanctionnés par une justice très mal en point. Le système judiciaire fait aussi l'objet d'une réforme pour des raisons très proches de celle de la police : faiblesse de l'expertise technique des magistrats, délais trop longs pour les instances et absence de suivi des jugements, comportements trop enclin à servir le pouvoir en place ou les justiciables les plus aisés.

A cela, il convient d'ajouter une concurrence entre un double système juridictionnel : le système de justice nationale dont la procédure est en français et dont le coût interdit toute action à des personnes pauvres à défaut d'aide judiciaire suffisante ; le système coutumier traditionnel en langue locale, utilisé essentiellement pour trancher les conflits liés à la propriété des terres, plus accessible à nombre de justiciables ${ }^{28}$.

La question toujours pendante concerne toutefois la mise en place des mécanismes de justice transitionnelle définis dans les Accords d'Arusha ${ }^{29}$. Ceux-ci prévoient un tribunal pénal international et une commission de vérité et de réconciliation. Les négociations ouvertes entre 2000 et 2005, correspondant à la période de transition n'ont pu aboutir.

Devant l'impasse politique, les Nations unies mandatent la mission Kalomoh pour faire des propositions. Le rapport remis en 2005 propose deux mécanismes différents pour amorcer le processus : l'instauration d'une commission vérité chargée d'enquêter sur les manquements graves aux droits de l'homme et crimes de masse et une chambre judiciaire spécialisée au sein du système pénal burundais pour juger des affaires. Le Conseil de sécurité charge son Bureau sur place de faciliter la négociation entre les différentes parties

27 Security Sector Reform Monitor Burundi, Cigi, November 2009, n 1, 12 p., p. 5.

28 Ibid., p. 9.

29 S.a., Le processus de justice de transition au Burundi; défis et perspectives, programme Afrique - ICTJ, 18 avril 2011. 
prenantes, sur cette base.

Le 2 novembre 2007, un accord-cadre créé un Comité tripartite Gouvernement burundais - société civile - Nation unies pour poursuivre le dialogue dans l'ensemble du pays, en y associant les organisations féminines. En dépit des attentes de la société civile, plusieurs sujets font obstacles à la création des nouvelles institutions :

- La question délicate de l'indépendance du procurer à l'égard du pouvoir politique gouvernemental ;

- La relation entre la commission vérité et réconciliation et le tribunal pénal ;

- Le champ de l'amnistie retenue ou non contre les auteurs de crimes de masse.

Le dialogue politique a été quasiment interrompu entre 2007 et 2010, date des dernières élections. L'ONU, soutenu par le Gouvernement belge et le SEAE ont cherché à relancer le processus ${ }^{30}$. En 2012, le Gouvernement a lancé la Commission vérité et réconciliation, mais elle n'avance guère.

\section{Les donateurs clés de la RSS burundaise}

Donateurs ET DOMAINES DE RÉFORME EN 2013

\begin{tabular}{|c|c|c|c|c|}
\hline & Armée & Police & Justice & Gouvernance \\
BNUB & $\mathrm{X}$ & $\mathrm{X}$ & $\mathrm{X}$ & $\mathrm{X}$ \\
\hline Pays-Bas & $\mathrm{X}$ & $\mathrm{X}$ & & $\mathrm{X}$ \\
\hline Allemagne & & $\mathrm{X}$ & & \\
\hline $\mathrm{BM} / \mathrm{FMI}$ & & & & $\mathrm{X}$ \\
\hline
\end{tabular}

Source : Security Sector Reform Resource Center

Le Bureau des Nations Unies au Burundi (BNUB) constitue l'organisme de conseil au gouvernement dans le renforcement de ses capacités institutionnelles. Il appuie le dialogue politique intercommunautaire, notamment sur les thèmes de l'impunité et du respect des droits de l'homme ${ }^{31}$ et suit les projets de réforme de la justice.

Les Néerlandais ont joué un rôle important dans la réforme de l'armée et de la police nationale, dès 2004. Le mémorandum actuellement en cours a été signé en 2009 et couvre la période jusqu'en 2017 sous le vocable de Netherlands-Burundi Security Sector Development Program (SSDP). Il inclut la sécurité publique, la défense et la gouvernance

30 Réponse à la question parlementaire n 5-1699 du 10 mars 2011 posée par la Sénatrice Olga Zihren au ministre belge des affaires étrangères.

31 Le mandate et les initiatives en cours sont accessibles sur le site suivant : http://bnub.unmissions.org/Default. aspx?tabid $=2962 \&$ language $=$ en - US 
de la RSS. Le programme est divisé en quatre phases de deux années, avec une première phase d'évaluation des besoins par secteur et d'orientation des programmes ${ }^{32}$.

L'Allemagne déploie un programme de mise à niveau des forces de police. Au Burundi, cela concerne la construction de commissariat dans les provinces, la constitution de capacités centrales (garages de véhicules), le travail sur des concepts et procédures de sécurité. Le plan comprend une première phase achevée 2008-2012 et une seconde en cours d'exécution pour 2012-2015 33 .

La Banque Mondiale et le Fond Monétaire International sont intervenus pour le financement du plan de DDR de 2004 à 2010. Ils se recentrent maintenant sur les activités de développement économique ${ }^{34}$.

\section{Rwanda : la RSS renforce l'appareil de contrôle policier et judiciaire de la société}

Le cas du Rwanda est quelque peu différent de celui du Burundi. Le processus de réforme initial prévu par les Accords d'Arusha du 4 août 1993 a été balayé par les conséquences du génocide de 1994 et la victoire des troupes du Front populaire rwandais (FPR).

L'enjeu pour le gouvernement consiste donc moins à rééquilibrer les appartenances communautaires au sein des institutions de sécurité, que de s'assurer de la prise en main durable de l'appareil d'Etat détruit pendant la guerre civile, en s'appuyant sur les forces militaires rwandaises issues du FPR (Armée populaire du Rwanda).

\footnotetext{
32_http://issat.dcaf.ch/Community-of-Practice/SSR-in-Practice/Countries-Regions/Burundi/Security-SectorDevelopment-Programme-for-Burundi-SSDP

33 Strengthening the capacity of the police in Burundi : http://www.giz.de/Themen/en/29822.htm 34 Les programmes actuellement en cours sont accessibles sur : http://web.worldbank.org/WBSITE/ EXTERNAL/NEWS/0,,contentMDK:23278406 menuPK:64256345 pagePK:34370 piPK:34424 theSit ePK:4607,00.html
} 


\begin{tabular}{|c|c|}
\hline Institution/Agency & Mandate/Function \\
\hline Rwandan Defence Forces & $\begin{array}{l}\text { Immediately after the } 1994 \text { genocide, the responsibility for maintaining } \\
\text { national security rested solely in the hands of the armed wing of the RPF. } \\
\text { the Rwandan Patriotic Army. Now, the Rwandan Defence Forces - largely } \\
\text { made up of former RPA soldiers - are responsible for the defense of the } \\
\text { country's territorial integrity and national sovereignty (Mironko and } \\
\text { Rurangwa, 2007: 216) }\end{array}$ \\
\hline Rwandan National Police (RNP) & $\begin{array}{l}\text { Established by Law No. } 09 / 2000 \text { of June } 16,2000 \text {, after the Government of } \\
\text { National Unity decided to combine the former Gendarmerie Nationale, which } \\
\text { was under the Ministry of Defense, with the former Communal Police under } \\
\text { the Ministry of Internal Affairs and the Judicial Police under the Ministry of } \\
\text { Justice (Kigali City, 2008). }\end{array}$ \\
\hline $\begin{array}{l}\text { Criminal Investigation Department } \\
\text { (CID) }\end{array}$ & $\begin{array}{l}\text { Responsible for coordinating criminal investigation activities. It is comprised } \\
\text { of six individual units, each with its own special tasks: Criminal } \\
\text { Investigations and Scientific Police (C.I.S.P). Anti-Narcotics Unit (A.N.U). } \\
\text { Economic and Financial Crimes (EFU), Interpol, Criminal Records Office } \\
\text { (C.R.O) and Administrative Unit (RNP, 2009a). }\end{array}$ \\
\hline Provincial Judicial Police & $\begin{array}{l}\text { Coordinates and supervises criminal investigations by judicial police officers } \\
\text { (RNP, 2009a). }\end{array}$ \\
\hline INTERPOL & $\begin{array}{l}\text { Also known as the National Central Bureau (NCB), it links the Rwandan } \\
\text { National Police to the Interpol General Secretariat in Lyon France and } \\
\text { National police forces of other member states of Interpol. It is responsible } \\
\text { for combating trans-national organized crimes such as drug trafficking. } \\
\text { human trafficking, crimes against women and children, cyber crimes, } \\
\text { money laundering, terrorism, stolen or forged travel documents, motor- } \\
\text { vehicle theft, etc. (RNP, } 2009 \text { c). }\end{array}$ \\
\hline $\begin{array}{l}\text { East African Police Chief's Committee } \\
\text { (EAPCCO) }\end{array}$ & $\begin{array}{l}\text { Ten East African member countries cooperate for the purpose of security } \\
\text { and capacity building (BIG, 2007). }\end{array}$ \\
\hline
\end{tabular}

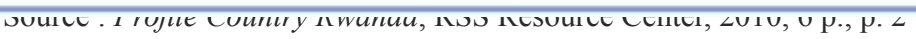

Le processus de RSS est largement financé par les donateurs internationaux, mais son architecture est conçue par le régime de manière autonome et très précise. L'appropriation ne pose aucun problème. Elle constitue la garantie de la survie du régime.

\section{La RSS militaire : s'ouvrir au peace-building régional en s'appuyant sur une armée ethniquement cohérente}

Le processus de DDR touche à sa fin. Engagé dès 1997 par la création d'une commission et d'un programme de démobilisation et de réintégration à l'initiative du Gouvernement rwandais, il a connu trois étapes :

- Une première étape (1997-2001) voit la démobilisation de 18600 excombattants ;

- La seconde étape (2002-2008) est engagée avec le soutien de la Banque 
mondiale, au sein du MDRP. Elle concerne 36730 ex-combattants ${ }^{35}$;

- La dernière étape (2009-2013) est conçue pour traiter les demandes résiduelles. Elle concerne 5500 ex-combattants n'appartenant pas aux forces armées rwandaises, dont 500 enfants-soldats et 4000 personnels des forces armées rwandaises. Un système de pécule au départ et d'assistance professionnelle par microcrédit permet de solder les comptes ${ }^{36}$.

Une attention particulière est accordée au retour des ex-combattants dans leur village ou communauté d'origine, en s'assurant qu'ils ne constituent plus une source de désordre avec leurs anciens voisins. C'est là où le système de surveillance policier et la justice de transition mis en place avec les Gacacas, jusqu'en 2010, autorise un contrôle local étroit.

Avec la fin du DDR, une nouvelle étape se profile misant sur la spécialisation d'une partie des forces au profit des opérations de maintien de la paix régionale. Le Rwanda dispose en effet d'un contingent de 3200 personnes au sein de la force hybride de l'Union africaine au Darfour (UNAMID). Il était donc logique que le « savoir-faire » rwandais dans le post-conflit puisse être montré et valorisé. Tel est l'objet de la création de l'Académie rwandaise de la paix. Sous le contrôle du ministère de la défense et avec le financement des Néerlandais, l'Académie aura pour vocation de constituer un centre d'expertise et de formation sur les conditions de sortie de conflit, les processus DDR et la RSS, l'entrainement aux meilleures pratiques dans les domaines de la prévention des conflits et du maintien de la paix.

\section{Les maillages croisés policier et judiciaire transitionnel : intimider la société civile}

La réforme de la police est menée avec autant de rigueur que celle des forces armées. Trois services ont été mis en place :

- La Rwandan National Police (RNP) a été créée le 12 juin 2000 en fusionnant l'ancienne gendarmerie (ministère de la défense), avec la police communale (ministère de l'intérieur) et la police judiciaire (ministère de la justice) ;

- Le Criminal Investigation Department (CID) est responsable des missions de police scientifique, de la lutte anti-drogue, de la criminalité organisée et financière, de la police spéciale anti-terroriste et les liaisons avec INTERPOL;

- La Provincial Judicial Police (PJP) supervise et coordonne les missions locales de police judiciaire.

35 S.a., The Rwanda Demobilization and Reintegration Program: Refections on the Reintegration of Ex-Combatants, MDRP Dissemination Note $\mathrm{n}^{\circ}$ 5, September-October 2008, 6 p., p.1.

36 Security Sector Reform Country Profile: Rwanda, CIGI, 2011, 6 p. 
Ces services sont alimentés en renseignements et informations d'environnement par le réseau des Community Partnerhip Committees : un maillage territorial dense organisé autour d'un groupe de surveillance du voisinage. Ce groupe composé d'un responsable, de sept adjoints et d'une milice locale rend compte des agissements de 500 à 1000 foyers en moyenne. Les comportements "déviants » sont ainsi immédiatement répertoriés et rapportés aux autorités compétentes ${ }^{37}$. Les enquêtes et instruction des dossiers liés au génocide demeurent, bien entendu, une priorité de l'ensemble des services.

Le Rwanda a dû faire face au problème fondamental de la reconstruction de son appareil judiciaire. Les contraintes sont d'abord liées à la dissolution du système précédent, discrédité par le génocide. Selon les statistiques du gouvernement actuel, il ne restait que 244 juges sur 750, 14 procureurs sur 87 en 1995. Il a fallu aussi faire face à l'épreuve du traitement judiciaire d'événements extraordinaires que sont les crimes de masse alors que 39 enquêteurs habilités sur 193 étaient encore disponibles ${ }^{38}$. Simultanément, le retour d'exil de milliers de Hutus et donc de personnes pouvant relever d'inculpations pour les événements de 1994, faisait planer le danger permanent des règlements de compte et des vengeances.

La situation se compliquait avec la création du Tribunal Pénal International pour le Rwanda, chargé de juger des concepteurs et exécutants des crimes de génocide, alors qu'il était évident que les moyens alloués ne suffiraient pas.

Ceci explique la constitution, à partir de 2002, d'une fonction juridictionnelle transitoire arcboutée sur les Gacacas : une assemblée locale et traditionnelle de médiation des conflits. Le recours aux Gacacas a permis, à partir de juges sélectionnés par les résidents, de créer un réseau de 9013 juridictions de premier niveau et de 1545 de niveau supérieur. La réforme de la justice de 2006 les intègre ensuite au système national jusqu'en 2011, date à laquelle les assemblées Gacacas sont dissoutes ${ }^{39}$. Le système est remarquablement intelligent et bon marché. Tout exilé de retour dans son village peut être mis accusation devant l'assemblée locale par un membre de la communauté et subir un procès. Ceci incite à une très grande prudence de la part des individus et un comportement loyal à l'égard du gouvernement. Le système atteint son plein rendement à partir de 2006. Selon les chiffres du ministère de la justice rwandais, 250000 assesseurs dans ces assemblées ont rendu près de 1,5 millions d'arrêts fin $2009^{40}$; soit des décisions concernant plus de $10 \%$ de la population.

37 Ibid., p. 3.

38 Ibid., p. 1.

39 Tackeuchi, S. Gacaca and DDR: The Disputable Record of State Building in Rwanda, JICA Research Paper $\mathrm{n}^{\circ}$ 32, July 2011, 38 p., pp. 13-14.

40 Security Sector Reform Country Profile: Rwanda, op. cit., p. 4. 


\title{
Les donateurs clés de la RSS rwandaise
}

Le drame vécu par le Rwanda a mobilisé de nombreux intervenants dont les organisations internationales.

\section{SSR Resources}

\begin{abstract}
Although the Government of Rwanda has placed an emphasis on local institutions and bodies for SSR, its current resources, infrastructure and human capacity are still insufficient. The sustainability of Rwanda's reform processes thus relies on a continued commitment from the international community in terms of resources and logistics.

UNDP: The "Democratic Governance Program", with a budget of US\$1 million, collaborates with the Rwandan Government to "build the capacity of the ministry of justice to review and draft laws and policies and sensitize the citizens on basic laws and individual rights; to build the capacities of the justice institutions in the areas of administration of justice, and law enforcement by building a strong judiciary plus an effective and well coordinated justice sector; promote crime prevention through encouraging community policing; strengthen peace building and reconciliation through support to Gacaca and increase access of justice to the people especially the most vulnerable by reinforcing legal aid mechanisms and mediation committees" (UNDP, 2010).
\end{abstract}

World Bank: Between 2008-2012 will give US\$20.8 million to build public sector capacities, specifically in public administration, law and justice (World Bank, 2010).

CIDA: Seeks to rebuild infrastructure, restore the systems of justice, and promote security in the Great Lakes Region. Through the Country Development Programming Framework (2005-2011), CIDA works to improve the ability of the justice system to support human and material resource management by installing information management systems and providing associated training (CIDA, 2010). The goal is to administer justice in a more efficient and transparent way.

UNAMIR: From its inception until its withdrawal in 1997, UNAMIR authorized over 5,000 troops and military support personnel, 320 military observers, 120 civilian police, approximately 160 international and local civilian staff and 56 UN volunteers to ensure security in Rwanda and assist in the training of the RNP (UNAMIR, 2010).

USAID: With US\$10.6 million devoted to Rwandan governance and democracy, USAID collaborates with the Government of Rwanda and other national bodies, including the National Unity and Reconciliation Commission, to develop national and local institutions of government, maintain security, promote reconciliation and strengthen the justice system (USAID, 2010).

Source : Profile Country Rwanda, RSS Resource Center, 2010, p. 5.

En 2013, on compte encore 10 donateurs principaux : 4 organisations internationales (Le fond mondial de lutte contre le SIDA, la tuberculose et le paludisme Le fond de développement africain - L'Union européenne) et 6 pays (Allemagne, Belgique, Canada, Etats-Unis, Pays-Bas, Royaume-Uni). Le Japon se trouve encore engagé au titre de contribution à un fond permanent de RSS.

Les projets principaux en cours concernent :

- $\quad$ Le Japon, financier de la Rwandan Peace Academy (RPA) avec le concours du Programme de développement des Nations unies (PNUD). L'ensemble du programme compte pour 3 millions de dollars (2012) et un peu moins d'un demi-million pour l'ONU ${ }^{41}$.

41 Support the Establishment of the Rwanda Peace Academy, http://www.rw.undp.org/content/rwanda/en/ home/operations/projects/democratic_governance/support-to-the-establishment-of-the-rwanda-peace-academy-rpa-/ 
- Le PNUD pour le programme de soutien de réforme de la justice ${ }^{42}$. Il s'agit pour l'essentiel de financer la formation technique de la magistrature ; d'instruire les administrations et les citoyens sur les droits et l'accès à la justice ; former les policiers aux différentes questions de la police de proximité au contact des différentes communautés ; combattre les idéologies génocidaires et promouvoir le respect entre différentes communautés ${ }^{43}$.

\section{La République Démocratique du Congo : la RSS otage de la guerre à l'Est}

Champs de bataille de deux guerres dans la région des Grands Lacs, la République Démocratique du Congo est en proie à une insécurité extérieure et intérieure dénoncée par les populations et les organisations non gouvernementales ${ }^{44}$.

En dépit d'une présence internationale forte, dont celles de L'ONU (MONUSCO) et de l'Union européenne (EUSEC RD Congo, EUPOL RD Congo), les structures de sécurité du pays, à commencer par les Forces armées de la République Démocratique du Congo (FARDC) sont incapables d'assurer les missions de contrôle du territoire. Le processus de RSS a connu de longs développements, mais se trouve entravé par l'absence de coordination entre les nombreuses parties prenantes.

Cette situation est utilisée par le pouvoir en place pour maintenir un statu quo servant des intérêts immédiats.

Un point important mérite d'être souligné : contrairement au Burundi et au Rwanda, la RDC est un pays qui subit la guerre sur son territoire et n'est donc pas en post-conflit, ni en « sortie de crise », situations normalement attendues pour mettre en œuvre une RSS.

\footnotetext{
42 Justice sector support programme 2008-2012 : Access to justice for all,the foundation of good governance and poverty reduction,

http://www.rw.undp.org/content/rwanda/en/home/operations/projects/democratic_governance/justice-sectorsupport-programme-2008-2012---access-to-justice-f.html

43 Un rapport complet sur l'architecture du programme est accessible dans : Justice Sector Support Programme 20082012 Access to justice for all, the foundation for good governance and poverty reduction, Government of the Republic of Rwanda / UNDP, 2012, 65 p.

44 S.a., République démocratique du Congo : prendre position sur la réforme du secteur de la sécurité, rapport collectif d'organisations non gouvernementales, 2011, 26 p.
} 


\section{La RSS militaire est entravée par des cycles répétitifs de DDR}

La RSS en RDC est inscrite dans plusieurs textes cadres fixant les termes du processus de paix dans les Grands Lacs :

- L'Accord de cessez-le-feu de Lusaka du 10 juillet 1999, signé entre la RDC, l'Angola, la Namibie, le Rwanda, l'Ouganda et le Zimbabwe, pour faire cesser les hostilités, auquel se joint le Mouvement de libération du Congo (MLC) au 1er août ;

- La résolution 1341 du 22 février 2001, par laquelle le Conseil de sécurité demande aux belligérants, entre autre chose, d'élaborer un plan de DDR ;

- La RSS est prévue dans l'Accord global inclusif de Sun City (RSA) du 17 décembre 2002, fixant le régime de la période de transition politique. L'accord dispose de la création d'un Conseil supérieur de défense responsable de la conception d'une armée « nationale intégrée et restructurée »... ;

- La constitution transitoire de 2003, qui décrit les missions des nouvelles FARDC.

\section{«Brassage » et « mixage »: les « mamelles » improbables de l'intégration}

Il faudra du temps, pendant la période de transition (2001-2006) pour entamer réellement le processus. Au départ des forces étrangères, il est convenu que les groupes armés constitueront, dans les régions où ils se trouvent, le corps des nouvelles institutions policière et militaire. Puis, progressivement, avec l'arrivée de donateurs nouveaux (les Belges en 2004 créant une première brigade intégrée à Kisangani), se met en place un réseau de centres de "brassage ", dans lesquels sont accueillis les ex-combattants pour intégrer les nouvelles brigades des FARDC. Celles-ci, au nombre théorique de 18, sont conçues pour " casser " les anciennes allégeances de la guerre et intégrer des personnels dans des unités déployées ensuite dans les différentes provinces du pays. Toutefois, les moyens feront défauts et de nombreuses brigades intégrées ne seront pas soutenues de manière adéquate. Cette absence de logistique aura pour conséquence de réduire la capacité opérationnelle de certaines d'entre-elles et d'obliger les soldats à vivre sur la population.

Une fois passées les élections de 2006, la guerre dans l'Est reprend cette fois contre le Congrès national pour la défense du peuple (CNDP) de Laurent Nkunda, remplacé ensuite par Bosco Ntaganda. Lors des négociations d'intégration du CNDP en 2007, les 7000 combattants sont "mixés » avec les FARDC présentes dans les Kivu. A la différence du brassage, le mixage maintien les groupes armés dans leur structure initiale. L'intégration s'opère au sein du commandement de la brigade entre les anciens commandants, disposant des grades d'officiers généraux dans la nouvelle armée. Ceci revient, en fait, à préserver les anciennes chaines de commandement en doublure de la nouvelle. Là aussi, faute de moyens suffisants, le gouvernement central n'a pu maintenir des revenus pour les nouvelles brigades, créant incidents et mutineries. Comme il était impossible de déplacer, les « mixés » hors du Kivu, leur région natale, ces unités ont continué à exploiter les richesses locales et constituées de fait une organisation politique et militaire hostile à Kinshasa. Il faudra attendre l'accord du 23 mars 2009, reconnaissant le CNDP comme parti politique, pour intégrer de nouveau les ex-CNDP.

En avril 2012, une nouvelle rébellion présentée comme une mutinerie contre les mauvaises conditions de vie des militaires, fait apparaître le Mouvement du 23 mars (M-23), créant une nouvelle rébellion avec la combinaison des déserteurs du CNDP et l'aide du Rwanda. Le cycle est reparti. 
Les autorités politiques vont ensuite produire plusieurs documents qui ne seront pas ou que très partiellement mis en application. En août 2005, le « Plan stratégique national pour l'intégration des forces armées ». Il fut suivi en 2007 par un «Schéma directeur de la réforme des forces armées » produit par l'état-major, mais écarté à la suite d'un désaccord avec le ministre de la défense. Deux plans le remplacèrent : un « Plan directeur de la réforme de l'armée » de 2008, repris en 2009 sous le titre de "Plan révisé de la réforme de l'armée », à la faveur d'un changement ministériel, avec l'assistance technique de l'EUSEC. En janvier 2010, un plan à long terme (15 ans) était présenté au financement de la communauté internationale $e^{45}$, mais avec de telles prétentions financières qu'il a très vite été jugé irréalisable.

Les dossiers de la RSS sont directement gérés par la Présidence, au sein du Conseil supérieur de la défense. Le Conseil dirige aussi les opérations dans l'Est, sans emprunter la chaîne de commandement des FARDC. Face à cette « hypercentralisation » présidentielle la communauté internationale se présente en désordre. Une première tentative de fédération des initiatives de RSS échoue avec l'instauration manquée du Comité de suivi des réformes de l'armée en 2008, suite au refus du gouvernement congolais. Une table ronde sur la RSS, activée la même année, et impliquant les grands donateurs avait pourtant conclus à la nécessité de cette coordination.

La MONUC s'est vue confiée la promotion de la RSS. En mai 2008, est créé le Bureau de coordination de la RSS avec un Coordinateur assurant le rôle de conseiller auprès du Représentant spécial du Secrétaire général. Les domaines de réformes concernent (résolution 1856) la composante militaire avec le soutien à la formation des brigades intégrées des FARDC ; la composante Etat de droit qui conseille le gouvernement sur le secteur de la justice et le renforcement des capacités judiciaires ; la composante police en charge de la formation de la Police nationale congolaise et de l'appui à la rédaction du cadre légal devant régir cette institution. Un Groupe de travail SSR sert de lieu d'échange avec les autres organisations internes de l'ONU (Programme des Nations Unies pour le Développement, le Programme International pour les Migrations) et des organisations partenaires (EUSEC et EUPOL RD Congo). Le Bureau devient celui de la MONUSCO à partir du 1er juillet 2010, quand la MONUC est rebaptisée par le Conseil de sécurité.

A l'exception de l'Union européenne, les contributions des donateurs sont essentiellement bilatérales et concernent l'équipement et l'entraînement de brigades pour déploiement immédiat, à la demande du Président congolais.

45 Boschoff, H. et al, Supporting SSR in the DRC: An Analysis of the Donors Approach, Netherlands Institute of International Relations, Clingendael, April 2010, 33 p., p. 19. 
L'Union Européenne : une tentative d'approche « heuristique » de la RSS.

L'Union dispose en 2013, de deux programmes de RSS encore en cours.

EUSEC RD Congo. La mission de conseil et d'assistance de l'UE en matière de RSS en RDC est créée par l'action commune 2005/355/PESC. Elle vise à soutenir la mise en cuvre du plan de réforme des Forces Armées de la République Démocratique du Congo (FARDC), notamment l'administration, les ressources humaines, la formation, la logistique, les droits de l'homme, le genre et la lutte contre les violences sexuelles, ainsi qu'au processus d'intégration des ex-combattants au sein des FARDC. Fin 2012, les 50 personnels présents sur zone étaient fournis par 14 Etats de l'Union. Le travail des équipes EUSEC a consisté à créer des capacités administratives et financières modernisées pour la gestion des payes des militaires (bancarisation) et la remise en fonctionnement des écoles de formation des officiers, sous-officier, de l'infanterie (Kitona), du commissariat (Kananga). Cela a permis de procéder à une identification biométrique des personnels et d'en établir un rôle aussi fiable que possible. La mission assiste aussi le ministère congolais de la défense dans l'étude et la définition des modèles d'armée entrant dans les différents plans de réforme et travaille sur un projet de statut du militaire.

EUPOL RD Congo a été autorisée par l'action commune 2007/405/0ESC du 12 juin 2007. Son mandat arrive à expiration le 30 septembre 2013. Elle remplace EUPOL Kinshasa, une mission civile de soutien et de formation de la police nationale dans la capitale congolaise pendant la période de transition politique s'achevant par les élections de 2006. Composé de 50 personnes fournies par 7 Etats de l'Union, EUPOL couvre quatre domaines principaux :

- Une mission de conseil pour la restructuration de la police nationale congolaise (PNC) dont les 100000 agents ne sont pas suffisamment formés pour les tâches techniques de police. En jeu, se trouve la constitution d'un corps de policiers intégré et multiethnique;

- Une mission d'amélioration du fonctionnement de la police criminelle et de son interaction avec la justice par le renforcement des capacités techniques d'enquête d'une part; la formation aux procédures pénales d'autre part ;

- Une mission de formation éthique des agents au respect des droits de l'Homme, des enfants et des femmes, notamment contre les abus sexuels et les viols.

Pratiquement, cela implique de mettre en place et de structurer une coordination interministérielle congolaise de manière à traiter l'ensemble des questions de sécurité publique. Ces contributions ont été utilisées par le législateur dans la loi du 11 août 2011 portant organisation et fonctionnement de la Police nationale congolaise : le nouveau cadre légal en vigueur.

Comme la MONUSCO, l'Union Européenne tente d'animer un groupe de coordination avec les Etats donateurs, sans grand succès.

Deux obstacles majeurs semblent s'opposer à une RSS profonde de l'armée, du moins selon les vœux des donateurs.

Tout d'abord, une absence de volonté politique de créer une administration efficace de la défense. Une garde présidentielle de 10000 à 15000 hommes, suffit à protéger Kinshasa, le régime et les centres principaux comme le Katanga. Il n'est pas nécessaire de développer une vraie administration centrale dispendieuse et en charge des brigades 
« brassées 》 ou « mixées » composées en partie d'anciens rebelles au régime. Il est encore moins utile de chercher à promouvoir des organes de contrôle parlementaires ou autres.

Ensuite, le coût d'une telle réforme est inconnu. Le gouvernement de RDC a pris l'habitude de privilégier les relations bilatérales avec les donateurs. Du point de vue congolais, il est plus habile de les mettre en concurrence ; du côté des donateurs, ceux-ci ont compris l'impossibilité d'une réforme " heuristique » des armées et se contentent de programme d'entraînement basique et de dons d'équipements, en contrepartie d'avantages divers. L'élaboration d'une planification budgétaire de programmation devient impossible. Ainsi, des Belges, des Américains, des Chinois, des Sud-Africains forment telle ou telle unité sans contacts directs, ni coordination ${ }^{46}$. Les unités formées étant ensuite consommées dans les conflits de l'Est ou abandonnées à leur sort dans les régions plus calmes, à défaut d'une logistique adéquate.

Finalement, l'obstacle essentiel à la RSS est constitué par la stratégie de « guerre populaire » d'inspiration maoïste, imposée par le Rwanda pour contrôler une partie des Kivu. Des groupes, dont les chefs peuvent être facilement stipendiés par un Etat parrain, s'établissent dans des zones clés ou résident leurs communautés. Ils disposent ainsi d'une possibilité de recrutement local et mettent en œuvre une administration parallèle des territoires, autorisant l'exploitation et la levée de ressources. Une partie sert à acheter les armes et les conseillers étrangers conférant des capacités militaires aussi importantes, voire supérieures à celles des FARDC. Comme ces dernières envoient sur place des brigades peu fiables et des soldats sans attache avec la région, elles sont vite usées. In fine, c'est par des négociations politiques que ces groupes sont ralliés par le Gouvernement congolais dans le cadre « d'accords de paix » prévoyant la reconnaissance politique du mouvement, l'inclusion des chefs dans de hauts postes administratifs et l'intégration des ex-combattants dans une nouvelle brigade des FARDC. Une fois l'accord engrangé, il suffit de quelques mois pour relancer un autre groupe mécontent et de réamorcer une insurrection locale, comme le montre le cas du M-23. Dans un tel environnement, le gouvernement de Kinshasa ne cherche pas autre chose que d'établir un rapport de force régional militaire. Le danger pour lui étant moins la rébellion locale que l'appui extérieur des forces armées organisées du Rwanda.

\section{La RSS policière n'est pas prioritaire : l'insécurité intérieure ne menace pas le gouvernement central}

A l'instar des forces armées, les institutions policières et judiciaires ont été largement laissées à l'abandon par le régime Mobutu. Les deux décennies de conflits qui ont suivi ont entravé toute possibilité sérieuse de réformes et considérablement diminué la capacité de la société civile à faire pression pour en exiger la remise en route.

46 Hendrickson, D. and Kasongo, M. Security Sector Reform in the DRC: Strategic Issues, Issue Paper $\mathrm{n}^{\circ}$ 4, Center on International Cooperation, African security sector network, 2010, 10 p. p. 5. 
La question de la réforme policière n'est pas initialement liée à la sécurité intérieure de la RDC, mais à l'organisation du processus de DDR (surveillance des centres de brassage), puis au suivi des processus électoraux devant marquer la fin de la période de transition.

Deux organismes tentent de concevoir un plan de réforme. Tout d'abord, le Groupe mixte de réflexion sur la réforme et la réorganisation de la police nationale congolaise (GMRRR). Il se réunit en 2006 avec un panel de 23 participants, dont 6 seulement appartiennent à la Police nationale congolaise (PNC), les autres relevant des différents donateurs. En 2007, un séminaire de consultation national est organisé, intégrant cette fois des représentants des institutions (Parlement, ministères) et de la société civile. C'est en cette occasion que sont définis les principes de la réforme à venir : unification des services de police judiciaire, des frontières, au sein de la PNC ; instauration d'un service civil, apolitique et républicain ${ }^{47}$.

C'est ensuite le Comité de suivi de la réforme de la police (CSRP) qui prend en compte l'étude et la mise en route de ces principes. Toutefois, les experts du Comité travailleront à l'extérieur de la PNC et les relations avec cette dernière seront très limitées. Le « Plan d'action de la réforme de la police » est issu des travaux du CSRP.

Les grands donateurs de la RSS policière : Royaume Uni, UE, MONUSCO-UNPOL.

Le Royaume-Uni finance un plan (2009-2014) baptisé : Security Sector Accountability Police Reform (SSAPR) d'un montant de 60 millions de Livres (environ 71,4 millions d'Euros 2013). Les efforts se concentrent dans les provinces du Kassaï, du Bas-Congo et du Sud Kivu. Les projets concernent la formation au leadership et à la gestion ; l'équipement et le service des systèmes de communication; des parcs de véhicules ; la coordination intérieure à la PNC; enfin la formation aux relations police locale et grand public.

Le rôle de l'Union européenne EUPOL RD Congo a déjà été présenté supra. Il n'est donc rappelé que pour mémoire.

La MONUC aujourd'hui MONUSCO-UNPOL dispose d'un effectif de 391 fonctionnaires de police et de gendarmerie auxquels s'ajoutent 1058 personnels composants des unités de police constituées (Formed Police Units - FPU). Ils sont fournis par 24 pays contributeurs (Egypte, Inde, Pakistan, Sénégal arment des FPU. L'état-major est situé à Kinshasa et des bureaux sont ouverts dans 11 provinces congolaises. Les personnels sont répartis par secteurs et sous-secteurs en soutien de la PNC. Les missions de l'UNPOL découlent de la Résolution 2053 du 12 juin 2012 et concernent les domaines principaux :

- La protection des civils avec un soutien à la PNC contre les agressions sexuelles et dans le cadre des tâches de maintien de l'ordre;

- La stabilisation et la consolidation de la paix par une activité de conseil au profit des autorités congolaises dans le renforcement et le pilotage des capacités institutionnelles. Un effort est consenti avec le programme Stratégies du gouvernement et de la communauté internationale pour la stabilisation et la reconstruction (STAREC) des zones sortant des conflits de l'Est de la RDC. Il s'agit pour l'essentiel de former et d'aider au déploiement de la PLN dans les zones libres de groupes armés. Elle suit l'intégration des ex-combattants du CNDP dans la police nationale ;

47 Boschoff et al, Supporting SSR in the DRC..., op. cit., p. 13. 
- Le soutien à la sécurisation des élections. Il s'agit essentiellement de mettre sur pied 6 bataillons de la Police d'Intervention Rapide (3 000 hommes) pour le maintien de l'ordre et la formation des polices locales (Groupement mobile d'intervention) à la gestion des événements électoraux. L'accent est mis sur le respect des droits de l'homme et de la procédure.

Au final, la réforme de la PNC s'est concentrée d'abord sur la reconstitution de l'institution policière et la création d'une bureaucratie fonctionnelle. Les problèmes de lutte contre la criminalité et les agressions sexuelles (souci premier de nombreux citoyens congolais) n'ont pas été pris en compte prioritairement par les donateurs. C'est aujourd'hui une préoccupation majeure dans la population.

Le financement du fonctionnement de la PNC demeure un souci constant. Or, la Présidence conserve la haute main sur le dossier, laissant au ministère de l'intérieur assez peu d'initiatives.

Finalement, les problèmes de criminalités ne sont pas perçus comme une menace létale contre le gouvernement actuel. La société civile étant encore fragile, il est peu probable qu'elle soit en mesure de s'organiser pour imposer ses propres priorités.

\section{La RSS judiciaire est entravée par la crainte d'une justice indépendante}

Une étude sociologique de terrain menée en 2010 à Lubumbashi, montrait l'émergence de stratégies de contournement par la population afin de régler le problème de l'accès à la justice : accords amiables, réparations volontaires à la victime, justice traditionnelle, voire justice privée par recours aux armes ou appel à la justice populaire (arrestation et lynchage d'un criminel) remplacent le recours aux tribunaux jugés incompétents, corrompus ou simplement inaccessibles ${ }^{48}$. Les conclusions indiquaient aussi la quasi incompréhension entre la population non francophone (une majorité dans le pays) et les institutions d'Etat et leurs procédures. Ce tableau dépeint bien la difficulté pratique de relever le secteur de la justice dans l'ensemble du pays.

Devant les difficultés, l'Union européenne, dès 2004, a joué un rôle essentiel dans le projet de réforme de la justice. La Commission a pris l'initiative de rassembler un ensemble de donateurs au sein d'un Comité mixte de justice (CMJ), en partenariat avec le ministère de la justice de RDC. En 2008, un «Plan d'action pour la réforme de la justice » est présenté. Il retient quatre thèmes prioritaires :

- Un accès égal pour tous à la justice ;

- La restauration d'un cadre institutionnel fonctionnel ;

- La lutte contre la corruption et l'impunité ;

- La promotion du respect des droits humains

48 Felices-Luna, M. « La Justice en République Démocratique du Congo : transformation ou continuité ?», Champ pénal, Volume VII, 2010 : accessible sur http://champpenal.revues.org/7827\#tocto2n3 
L'ensemble des donateurs couvre à peu près tout l'édifice juridictionnel, y compris le système pénitentiaire. Le montant global des contributions représentait un montant de 150 millions de dollars (2010), englobant 10 programmes construits autour de 48 projets $^{49}$.

Cependant, en raison de la superficie du pays, il est impossible d'assurer une stratégie intégrale de restauration de toutes les institutions judiciaires, pénales et pénitentiaires. Pour cette raison, les donateurs ont décidé de se concentrer dans des zones géographiques prioritaires. Il y donc bien une approche « intégrale », mais à l'échelle locale comme le montre le programme REJUSCO.

REJUSCO : rétablir la justice dans l'Est de la RDC.

Le programme de la Restauration de la Justice à l'Est de la RDC, REJUSCO est une initiative prise par la Commission européenne pour la période 2007-2010, en partenariat avec les coopérations britannique, belge et néerlandaise. Après avoir rétabli une administration judiciaire à Bunia, le projet de 11,5 millions d'Euros (9ème FED) a consisté à étendre l'initiative au Nord Kivu, Sud Kivu et l'Ituri. L'ensemble a été mis en place par la Coopération technique belge. La réimplantation des administrations (réfection des bâtiments) s'accompagnait d'un financement pour permettre un accès égal à la justice et assurer le traitement rapide des dossiers. Les bureaux disséminés à Goma, Bukavu et Bunia ont été fermés au printemps 2010.

Le balancement des ressources au profit du niveau local, limite les évolutions des institutions nationales. Ces dernières sont victimes des retards enregistrés dans le vote des lois de réforme devant le Parlement. Les réformes nationales sont surtout victimes d'un sous-financement organisé par le pouvoir central. Ainsi, en 2010, la part du budget de la justice dans le budget de l'Etat était limitée à $0,22 \%{ }^{50}$. Les grands défis de la lutte contre la corruption et l'établissement d'un corps de magistrats indépendants sont donc retardés d'autant. Cette déshérence nationale ne menace donc pas les intérêts actuellement en place.

\section{Conclusion}

La réforme du secteur de la sécurité dans l'Afrique des Grands Lacs offre des exemples très illustratifs de la difficulté d'implanter localement des concepts extérieurs au Continent.

Premier enseignement : ces processus, requérant de nombreuses compétences techniques, sont avant tout des machines politiques extrêmement fragiles. Chacun des bénéficiaires l'intègre dans sa stratégie de survie ; c'est une évidence. Mais les donateurs s'en servent aussi souvent comme instrument d'influence, voire de monnaie d'échange pour tirer des avantages pratiques des bénéficiaires. Cette confrontation des intérêts ne facilite pas l'approche globale et intégrée recommandée par les spécialistes du domaine. Elle l'interdit même souvent, de fait.

49 Boschoff et al, Supporting SSR in the DRC, op. cit., p. 5.

50 Ibid., p. 10. 
Second enseignement: il n'existe pas d'exemples pratiques d'exercice de « réformes heuristiques » des systèmes de sécurité dans la région. L'importance des appareils de force militaire et de police, parfois la justice concentrent l'essentiel des attentions et des programmes, en termes de financement et de moyens. Et encore, s'aperçoit-on rapidement que ce sont les budgets de DDR qui constituent la majeure partie des dépenses, limitant souvent les ressources disponibles pour les autres domaines. A cet égard, les efforts consentis avec la MDRP montrent l'étendue maximale consentie par la communauté internationale. De tels programmes ne sont pas reproductibles dans le domaine encore plus complexe des RSS.

Troisième enseignement : contrairement aux craintes exprimées par de nombreux auteurs sur le risque de défaut d'appropriation des acteurs locaux, il est évident que c'est l'inverse qui se produit. Dans le cas du Burundi et du Rwanda, l'instrument RSS est véritablement intégré aux dynamiques politiques. Au Burundi, les négociations autour des réformes des institutions de sécurité démontrent un choix de concertation, même difficile entre les partenaires / adversaires politiques. Au Rwanda, la RSS est utilisée par le Gouvernement comme un outil au service de la "Tutsisation » de l'Etat, dans le but d'entraver tout risque de rébellion interne et de maximiser un contrôle social vital pour la survie du régime. En RDC, la Présidence ne retient de la réforme que ce qui l'intéresse : c'est-à-dire des moyens additionnels pour contenir les désordres de l'Est, en complément du dispositif de la MONUSCO, tandis que l'essentiel de l'appareil militaire utile, la Garde présidentielle, reste sous contrôle stricte de Kinshasa. Dans les trois cas, les moyens alloués par l'extérieur sont pilotés de manière intelligente par les gouvernements bénéficiaires.

Oui, les Grands Lacs constituent bien aujourd'hui, le principal laboratoire de la RSS. Mais les produits qui chauffent dans ses cornues ont des caractéristiques propres que les initiateurs de ces programmes n'avaient pas prévues. Or, après tout, c'est bien à cela que sert un laboratoire ! 